\title{
Rigid bronchoscopy for airway foreign body removal - Complication related factors
}

\author{
F.V. Marques ${ }^{1}$, A. Sampaio ${ }^{1}$, T. Lapa ${ }^{1,2}$, G. Paiva ${ }^{1,3}$ \\ 1 - Coimbra Hospital and University Centre, Coimbra, Portugal \\ 2 - University of Beira Interior, Portugal, Faculty of Health Sciences, Covilhã, Portugal \\ 3 - Paediatric Hospital - Coimbra Hospital and University Centre, Coimbra, Portugal
}

Background and Goal of Study: Airway foreign body aspiration is a risk in children, especially under the age of 3 . Early removal of the foreign body by rigid bronchoscopy is crucial, but complications during the procedure may develop $(1,2)$. One of the most frequent complications is hypoxemia (3). The goal of this study was to evaluate the development of complications during the anesthetic management of airway foreign body aspiration and also compare groups in what concerns to intraprocedural complications.

Materials and Methods:

- Type of study: retrospective, observational;

- Population: children who underwent rigid bronchoscopy (urgent and elective) under general anesthesia;

- Inclusion criteria: age $>0$ and $<18$ years old, and complete records of the study variables;

- Data analysis: SPSS using the chi-square test and Mann-whitney test,

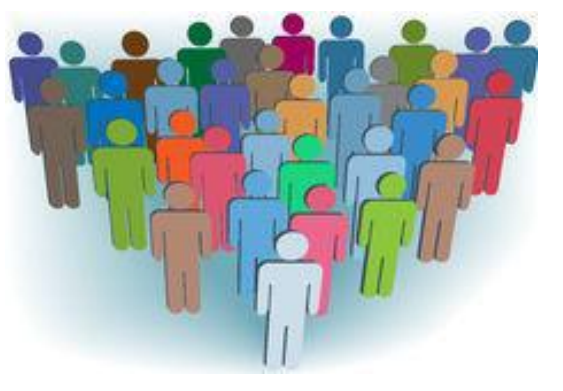

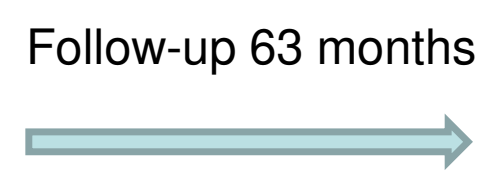

36 patients

\section{Complications}

No intercurrences

Results and Discussion:

- 36 patients were included in the study with a mean age of 4,2 years;

- Among the 36 children, 2 developed complications, including hypoxemia $(\mathrm{N}=1)$ and cardiac arrest $(\mathrm{N}=1)$;

- There were no other reported incidents in the remaining patients;

- Group comparisons are shown in table 1 and 2.

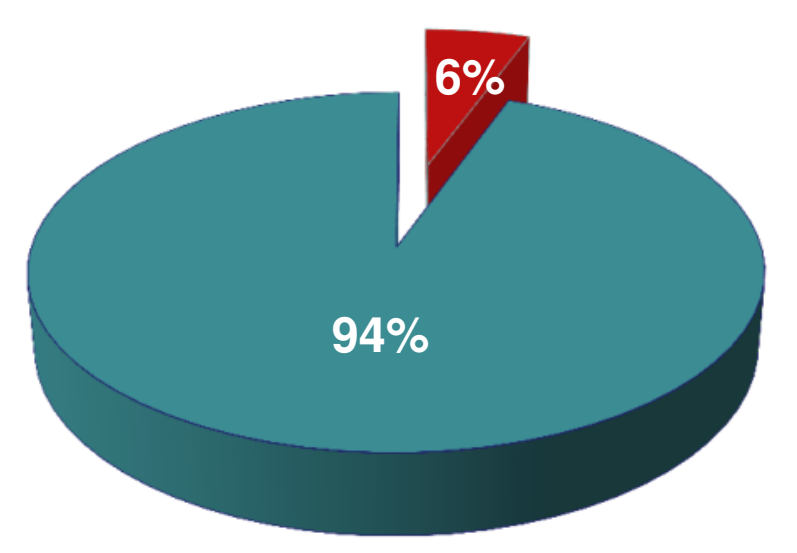

- Complications

$\square$ Without intercurrences

\begin{tabular}{|c|c|c|c|}
\hline \multirow{2}{*}{ Age } & \multicolumn{2}{|c|}{ Median 2,50 years old } & \multirow{2}{*}{0,667} \\
\hline \multirow{2}{*}{ Gender } & Male & $21(58,3 \%)$ & \multirow{2}{*}{0,806} \\
\cline { 2 - 3 } & Female & $15(41,7 \%)$ & \\
\hline Weigh & Median 12,00 kg & 0,188 \\
\hline \multirow{2}{*}{ ASA physical status } & I & $24(66,7 \%)$ & \multirow{2}{*}{0,00} \\
\cline { 2 - 3 } & II & $11(30,5 \%)$ & \\
\hline \multirow{2}{*}{ Procedure } & IV & $1(2,8 \%)$ & \multirow{2}{*}{0,129} \\
\cline { 2 - 3 } & Urgent & $31(86,1 \%)$ & \multirow{2}{*}{0,622} \\
\hline
\end{tabular}

Table 1

Conclusion:

- Airway management is a concern during bronchoscopy for airway foreign body aspiration;

- Our sample showed a low rate of intraprocedural complications. It can be related to the experience of the multidisciplinary team that is activated in these situations;

- The development of complications appeared to be related to the ASA physical status and the ventilation mode used;

- Subsequent studies with larger samples are needed to draw further conclusions.

References:

1) Chai $J$,

Fidkowski. "A retrospective study of anesthesia during rigid bronchoscopy for airway foreign body removal in children: propofol and sevoflurane with spontaneous ventilation "

Fidkowski CW, et al. "The anesthetic considerations of tracheobronchial foreign bodies in children: a literature review of 12,979 cases." Anesth Analg 111.4 (2010:):1016-25.
Shen, Xia, et al. "Propofol-remifentanil intravenous anesthesia and spontaneous ventilation for airway foreign body removal in children with preoperative respiratory impairm 